\title{
Cyclic homology with coefficients
}

\author{
D. Kaledin* \\ To Yu. I. Manin, the founder, on the occasion of his 70-th birthday
}

\section{Contents}

1 Recollection on cyclic homology.

2 Cyclic bimodules.

3 Gauss-Manin connection.

4 Categorical approach. $\quad 16$

5 Discussion 25

\section{Introduction}

Ever since it was discovered in 1982 by A. Connes [C1] and B. Tsygan [Ts], cyclic homology occupies a strange place in the realm of homological algebra. Normally in homological algebra problems, one expects to start from some data, such e.g. a topological space $X$, then construct some abelian category, such as the category of sheaves on $X$, and then define the cohomology of $X$ by computing the derived functors of some natural functor, such as e.g. the global sections functor $\Gamma(X,-)$. Admittedly, this is a modern formulation, but it had certainly been current already in 1982. Cyclic homology starts with an associative algebra $A$, and defines its homology groups $H C$. $(A)$, but there are absolutely no derived functors in sight. Originally, $H C$. $(A)$ were defined as the homology of an explicit complex, - which anyone trained to use triangulated categories cannot help but take as an insult. Later A. Connes

*Partially supported by CRDF grant RUM1-2694-MO05. 
C2 improved on the definition by introducing the abelian category of socalled cyclic vector spaces. However, the passage from $A$ to its associated cyclic vector space $A_{\#}$ is still done by an explicit ad hoc formula. It is as if we were to know the bar-complex which computes the homology of a group, without knowing the definition of the homology of a group.

This situation undoubtedly irked many people over the years, but to the best of my knowledge, no satisfactory solution has been proposed, and it may not exist - indeed, many relations to the de Rham homology notwithstanding, it is not clear whether cyclic homology properly forms a part of homological algebra at all (to the point that e.g. in [FT] the word "homology" is not used at all for $H C$. $(A)$, and it is called instead additive $K$-theory of $A$ ). In the great codification of homological algebra done in GM1, cyclic homology only appears in the exercises. This is not surprising, since the main unifying idea of [GM1 is the ideology of "linearization": homological algebra linearizes geometry, just as functional analysis used to do 50 years ago; triangulated categories and adjoint functors are modern-day versions of Banach spaces and adjoint linear operators. This has been an immensely successful and clarifying point of view, in general, but $H C$. $(A)$ sticks out on a complete tangent - there is simply no natural place for it in this framework.

This paper arouse as one more attempt to propose a solution to the difficulty - to find a natural triangulated category where $H C .(-)$ would be able to live with a certain level of comfort (and with all the standard corollaries such as the notion of cyclic homology with coefficients, the ability to compute cyclic homology by whatever resolution is convenient, not just the bar resolution, and so on).

In a sense, our attempt has been successful: we define a triangulated category which can serve as the natural "category of coefficients" for cyclic homology of an algebra $A$, and we prove the comparison theorem that shows that when the coefficients are trivial, the new definition of cyclic homology is equivalent to the old one. In fact, the algebra $A$ enters into the construction only through the category $A$-bimod of $A$-bimodules; we also show how to generalize the construction so that $A$-bimod is replaced with a more general tensor abelian category $\mathcal{C}$.

From a different point of view, though, out attempt failed miserably: the correspondence $A \mapsto A_{\#}$, being thrown out of the window, immediately returns through the door in a new and "higher-level disguise": it is now applied not to the algebra $A$, but to the tensor category $\mathcal{C}=A$-bimod. Then in practice, the freedom to choose an arbitrary resolution to compute the derived functors leads, in our approach to $H C$. (-), to complexes which 
are even larger than the original complex, and at some point the whole exercise starts to look pointless.

Still, we believe that, all said and done, some point can be found, and some things are clarified in our approach; one such thing is, for instance, the version of Gauss-Manin connection for cyclic homology discovered by E. Getzler [Ge]. Beside, we do propose a definition of cyclic homology which makes sense for a general tensor category; and in some particular questions, even the computations can be simplified. As for the presence of the $A_{\#^{-}}$ construction, this might be in the nature of things, after all - not a bug of the theory, but a necessary feature. However, we best leave it to the reader to be the judge.

The paper is organized as follows. In Section 1 we recall A. Connes' second definition of cyclic homology which uses the cyclic category $\Lambda$; we also recall some facts about homology of small categories that we will need. We have tried to give only the absolute minimum - the reader not familiar with the material will have to consult the references. In Section 2 we introduce our main object: the notion of a cyclic bimodule over an associative algebra $A$, and the derived category of such bimodules. We also introduce cyclic homology $H C .(A, M)$ with coefficients in a cyclic bimodule $M$. In Section 3 we give a very short derivation of the Gauss-Manin connection; strictly speaking, the language of cyclic bimodules is not needed for this, but we believe that it shows more clearly what is really going on. In Section 4, we show how to replace the category $A$-bimod everywhere with a more general tensor abelian category $\mathcal{C}$. Section 5 is a postface, or a "discussion" (as they do in medical journals) - we discuss some of the further things one might (and should) do with cyclic bimodules, and how to correct some deficiencies of the theory developed in Sections 2 and 4.

Acknowledgements. In the course of this work, I have benefited greatly from discussions with A. Beilinson, E. Getzler, V. Ginzburg, A. Kuznetsov, N. Markarian, D. Tamarkin, and B. Tsygan. I am grateful to Northwestern Univeristy, where part of this work was done, and where some of the results were presented in seminars, with great indulgence from the audience towards the unfinished state they were in. And, last but not least, it is a great pleasure and a great opportunity to dedicate the paper to Yuri Ivanovich Manin on his birthday. Besides all the usual things, I would like to stress that it is the book [GM1, - and GM2], to a lesser extent - which shaped the way we look at homological algebra today, at least "we" of my generation and of Moscow school. Without Manin's decisive influence, this paper certainly 
would not have appeared (as in fact at least a half of the papers I ever wrote).

\section{Recollection on cyclic homology.}

We start by recalling, extremely briefly, A. Connes' approach to cyclic homology, which was originally introduced in [C2] (for detailed overviews, see e.g. [L, Section 6] or [FT, Appendix]; a brief but complete exposition using the same language and notation as in this paper can be found in $\mathrm{Ka}$, Section 1]).

Connes' approach relies on the technique of homology of small categories. Fix a base field $k$. Recall that for every small category $\Gamma$, the category $\operatorname{Fun}(\Gamma, k)$ of functors from $\Gamma$ to the category $k$-Vect of $k$-vector spaces is an abelian category with enough projectives and enough injectives, with derived category $\mathcal{D}(\Gamma, k)$. For any object $E \in \operatorname{Fun}(\Gamma, k)$, the homology $H_{.}(\Gamma, E)$ of the category $\Gamma$ with coefficients in $E$ is by definition the derived functor of the direct limit functor

$$
\lim _{\vec{\Gamma}}: \operatorname{Fun}(\Gamma, k) \rightarrow k-\text { Vect }
$$

Analogously, the cohomology $H^{\bullet}(\Lambda, E)$ is the derived functor of the inverse limit $\underset{\leftarrow}{\lim }$. Equivalently,

$$
H^{\bullet}(\Gamma, E)=\operatorname{Ext}^{\bullet}(k, E),
$$

where $k \in \operatorname{Fun}(\Gamma, k)$ is the constant functor (all objects in $\Gamma$ go to $k$, all maps go to identity). In particular, $H^{\bullet}(\Gamma, k)$ is an algebra. For any $E \in$ Fun $(\Gamma, k)$, the cohomology $H^{\bullet}(\Gamma, E)$ and the homology $H_{\bullet}(\Gamma, E)$ are modules over $H^{\bullet}(\Gamma, k)$.

We also note, although it is not needed for the definition of cyclic homology, that for any functor $\gamma: \Gamma^{\prime} \rightarrow \Gamma$ between two small categories, we have the pullback functor $\gamma^{*}: \operatorname{Fun}(\Gamma, k) \rightarrow \operatorname{Fun}\left(\Gamma^{\prime}, k\right)$, and for any $E \in \operatorname{Fun}(\Gamma, k)$, we have natural maps

$$
H_{\bullet}\left(\Gamma^{\prime}, \gamma^{*} E\right) \rightarrow H_{\bullet}(\Gamma, E), \quad H^{\bullet}(\Gamma, E) \rightarrow H^{\bullet}\left(\Gamma^{\prime}, \gamma^{*} E\right) .
$$

Moreover, the pullback functor $\gamma^{*}$ has a left adjoint $\gamma !: \operatorname{Fun}\left(\Gamma^{\prime}, k\right) \rightarrow$ $\operatorname{Fun}(\Gamma, k)$ and a right-adjoint $f_{*}: \operatorname{Fun}\left(\Gamma^{\prime}, k\right) \rightarrow \operatorname{Fun}(\Gamma, k)$, known as the left and right Kan extensions. In general, $f_{!}$is right-exact but it need not be left-exact. We will need one particular case where it is exact. Assume given 
a covariant functor $V: \Gamma \rightarrow$ Sets from a small category $\Gamma$ to the category of sets, and consider the category $\Gamma^{\prime}$ of pairs $\langle[a], v\rangle$ of an object $[a] \in \Gamma$ and an element $v \in V([a])$ (maps in $\Gamma^{\prime}$ are those maps $\gamma:[a] \rightarrow\left[a^{\prime}\right]$ which send $v \in V([a])$ to $v^{\prime} \in V\left(\left[a^{\prime}\right]\right)$. Such a category is known as a discrete cofibration over $\Gamma$ associated to $V$, see $[\mathrm{Gr}$. Then the Kan extension $f$ ! associated to the forgetful functor $f: \Gamma^{\prime} \rightarrow \Gamma,\langle[a], v\rangle \mapsto[a]$ is exact, and is easy to compute: for any $E \in \operatorname{Fun}\left(\Gamma^{\prime}, k\right)$ and $[a] \in \Gamma$, we have

$$
f_{!} E([a])=\bigoplus_{v \in V([a])} E(\langle[a], v\rangle) .
$$

Moreover, for any $E \in \operatorname{Fun}(\Gamma, k)$, this imediately gives the projection formula:

$$
f_{!} f^{*} E \cong E \otimes F_{!} k,
$$

where, as before, $k \in \operatorname{Fun}\left(\Gamma^{\prime}, k\right)$ stands for the constant functor.

For applications to cyclic homology, one starts with introducing the cyclic category $\Lambda$. This is a small category whose objects $[n]$ are numbered by positive integers $n \geq 1$. One thinks of an object $[n]$ as a circle $S^{1}$ with $n$ distinct marked points; we denote the set of these points by $V([n])$. The set of maps $\Lambda\left(\left[n^{\prime}\right],[n]\right)$ from $\left[n^{\prime}\right]$ to $[n]$ is then the set of homotopy classes of continuous maps $f: S^{1} \rightarrow S^{1}$ such that

- $f$ has degree 1 , sends marked points to marked points, and is nondecreasing with respect to the natural cyclic order on $S^{1}$ (that is, if a point $a \in S^{1}$ lies between points $b$ and $c$ when counting clockwise, then the same is true for $f(a), f(b)$ and $f(c))$.

In particular, we have $\Lambda([1],[n])=V([n])$. This topological description of the cyclic category $\Lambda$ is easy to visualize, but there are also alternative combinatorial descriptions (e.g. [GM1, Exercize II.1.6], [L, Section 6], or [FT, A.2], retold in [Ka, Section 1.4]). All the descriptions are equivalent. Objects in $\operatorname{Fun}(\Lambda, k)$ are usually called cyclic vector spaces.

The cyclic category $\Lambda$ is related to the more familiar simplicial category $\Delta^{o p p}$, the opposite to the category $\Delta$ of finite non-empty linearly ordered sets. To understand the relation, consider the discrete cofibration $\Lambda_{[1]} / \Lambda$ associated to the functor $V: \Lambda \rightarrow$ Sets - equivalently, $\Lambda_{[1]}$ is the category of objects $[n]$ in $\Lambda$ eqipped with a map $[1] \rightarrow[n]$. Then it is easy to check that $\Lambda_{[1]}$ is equivalent to the $\Delta^{o p p}$. From now on, we will abuse the notation and identify $\Lambda_{[1]}$ and $\Delta^{o p p}$. We then have a natural projection $\Delta^{o p p}=\Lambda_{[1]} \rightarrow \Lambda$, $\langle[n], v\rangle \mapsto[n]$, which we denote by $j: \Delta^{o p p} \rightarrow \Lambda$. 
For any cyclic $k$-vector space $E \in \operatorname{Fun}(\Lambda, k)$, we have its restriction $j^{*} E \in$ $\operatorname{Fun}\left(\Delta^{o p p}, E\right)$, a simplicial vector space. One defines the cyclic homology $H C$. $(E)$ and the Hochschild homology $H H$. of $E$ by

$$
H C .(E) \stackrel{\text { def }}{=} H_{\bullet}(\Lambda, E), \quad H H_{\bullet}(E) \stackrel{\text { def }}{=} H_{\bullet}\left(\Delta^{o p p}, j^{*} E\right) .
$$

By (1.1), we have a natural map $H H_{.}(E) \rightarrow H C .(E)$ (moreover, since $j: \Delta^{o p p} \rightarrow \Lambda$ is a discrete cofibration, the Kan extension $j$ ! is exact, so that we have $H H_{\text {. }}(E) \cong H C \cdot\left(j_{!} j^{*} E\right)$, and the natural map is induced by the adjunction map $j ! j^{*} E \rightarrow E$ ). It has been shown by $\mathrm{A}$. Connes that this map fits into a long exact sequence

$$
H H_{\bullet}(E) \longrightarrow H_{\bullet}(E) \stackrel{u}{\longrightarrow} H C_{\bullet-2}(E) \longrightarrow \text {. }
$$

Here the map $u$ is the so-called periodicity map on $H C$. $(E)$ : one shows that the algebra $H^{\bullet}(\Lambda, k)$ is isomorphic to the polynomial algebra $k[u]$ in one generator $u$ of degree 2 , and the periodicity map on homology is simply the action of this generator. This allows to define a third homological invariant, the periodic cyclic homology HP. $(E)$ - to do it, one inverts the periodicity map.

Definition 1.1. For any cyclic $k$-vector space $E \in \operatorname{Fun}(\Lambda, k)$, the periodic cyclic homology of $E$ is defined by

$$
H P .(E)=\lim _{\leftarrow} \cdot H C .(E),
$$

where $\lim _{\leftarrow}{ }^{\bullet}$ denotes the derived functor of the inverse limit $\lim _{\leftarrow}$.

Assume now given an associative unital algebra $A$ over $k$. To define its cyclic homology, we associate to $A$ a canonical cyclic vector space $A_{\#}$ in the following way. We set $A_{\#}([n])=A^{\otimes V([n])}$, the tensor product of $n$ copies of the vector space $A$ numbered by marked points $v \in V([n])$. Then for any map $f \in \Lambda\left(\left[n^{\prime}\right],[n]\right)$, we define

$$
A_{\#}(f)=\bigotimes_{v \in V([n])} m_{f^{-1}(v)}: A^{\otimes V\left(\left[n^{\prime}\right]\right)}=\bigotimes_{v \in V([n])} A^{\otimes f^{-1}(v)} \rightarrow A^{\otimes V([n])},
$$

where for any linearly ordered finite set $S, m_{S}: A^{\otimes S} \rightarrow A$ is the canonical multiplication map induced by the associative algebra structure on $A$ (and if $S$ is empty, we set $A^{\otimes S}=k$, and $m_{S}$ is the embedding of the unity). This is obviously compatible with compositions, and it is well-defined since for any $v \in V([n])$, its preimage $f^{-1} \subset V([m])$ carries a natural linear order induced by the orientation of the circle $S^{1}$. 
Definition 1.2. For any associative unital algebra $A$ over $k$, its Hochschild, cyclic and periodic cyclic homology $H H_{\bullet}(A), H C .(A), H P .(A)$ is defined as the corresponding homology of the cyclic $k$-vector space $A_{\#}$ :

$$
H H_{\bullet}(A) \stackrel{\text { def }}{=} H H_{\bullet}\left(A_{\#}\right), \quad H C .(A) \stackrel{\text { def }}{=} H C .\left(A_{\#}\right), \quad H C .(P) \stackrel{\text { def }}{=} H P .\left(A_{\#}\right) .
$$

\section{Cyclic bimodules.}

Among all the homology functors introduced in Definition 1.2. Hochschild homology is the most accesible, and this is because it has another definition: for any associative unital algebra $A$ over $k$, we have

$$
H_{\text {. }}=\operatorname{Tor}_{A^{o p p} \otimes A}(A, A) \text {, }
$$

where Tor ${ }^{\bullet}$ is taken over the algebra $A^{\text {opp }} \otimes A$ (here $A^{\text {opp }}$ denotes $A$ with the multiplication taken in the opposite direction).

This has a version with coefficients: if $M$ is a left module over $A^{o p p} \otimes A$, - in other words, an $A$-bimodule, - one defines Hochschild homology of $A$ with coefficients in $M$ by

$$
H H_{\bullet}(A, M)=\operatorname{Tor}_{A^{o p p} \otimes A}(M, A) .
$$

The category $A$-bimod of $A$-bimodules is a unital (non-symmetric) tensor category, with tensor product $-\otimes_{A}-$ and the unit object $A$. Hochschild homology is a homological functor from $A$-bimod to $k$-Vect.

To obtain a small category interpretation of $H H .(A, M)$, one notes that for any $n, n^{\prime} \geq 0$, the $A$-bimodule structure on $M$ induces a multiplication map

$$
A^{\otimes n} \otimes M \otimes A^{\otimes n^{\prime}} \rightarrow M .
$$

Therefore, if to any $\langle[n], v\rangle \in \Delta^{o p p}$ we associate the $k$-vector space

$$
M_{\#}^{\Delta}([n])=M \otimes A^{\otimes(V([n]) \backslash\{v\})},
$$

with $M$ filling the place corresponding to $v \in V([n])$, then (1.5) make perfect sense for those maps $f:\left[n^{\prime}\right] \rightarrow[n]$ which preserve the distinguished points. Thus to any $M \in A$-bimod, we can associate a simplicial $k$-vector space $M_{\#}^{\Delta} \in \operatorname{Fun}\left(\Delta^{o p p}, k\right)$. In the particular case $M=A$, we have $A_{\#}^{\Delta}=j^{*} A_{\#}$.

Lemma 2.1. For any $M \in A$-bimod, we have a canonical isomorphism

$$
H H_{\bullet}(A, M) \cong H_{\bullet}\left(\Delta^{o p p}, M_{\#}^{\Delta}\right) .
$$


Proof. It is well-known that for any simplicial $k$-vector space $E$, the homology $H_{.}\left(\Delta^{o p p}, E\right)$ can be computed by the standard complex of $E$ (that is, the complex with terms $E([n])$ and the differential $d=\sum_{i}(-1)^{i} d_{i}$, where $d_{i}$ are the face maps). In particular, $H_{0}\left(\Delta^{o p p}, M_{\#}^{\Delta}\right)$ is the cokernel of the map $d: A \otimes M \rightarrow M$ given by $d(a \otimes m)=a m-m a$. The natural projection $M \rightarrow M \otimes_{A^{o p p} \otimes A} A$ obviously factors through this cokernel, so that we have a natural map

$$
\rho_{0}: H_{0}\left(\Delta^{o p p}, M_{\#}^{\Delta}\right) \rightarrow H H_{0}(A, M) .
$$

Both sides of (2.4) are homological functors in $M$, and $H H .(A, M)$ is a universal homological functor (=the derived functor of $H H_{0}(A, M)$ ); therefore the map $\rho_{0}$ extends to a map $\rho_{\text {. }}: H_{\bullet}\left(\Delta^{o p p}, M_{\#}^{\Delta}\right) \rightarrow H_{\bullet}(A, M)$. To prove that $\rho$. is an isomorphism for any $M$, it suffices to prove it when $M$ is free over $A^{o p p} \otimes A$, or in fact, when $M=A^{o p p} \otimes A$. Then on one hand, $H H_{0}(A, M)=A$, and $H H_{i}(A, M)=0$ for $i \geq 1$. And on the other hand, the standard complex associated to the simplicial $k$-vector space $\left(A^{\text {opp }} \otimes A\right)_{\#}^{\Delta}$ is just the usual bar resolution of the diagonal $A$-bimodule $A$.

It is more or less obvious that for an arbitrary $M \in A$-bimod, $M_{\#}^{\Delta}$ does not extend to a cyclic vector space - in order to be able to define $H C .(A, M)$, we have to equip the bimodule $M$ with some additional structure. To do this, we want to use the tensor structure on $A$-bimod. The slogan is the following:

- To find a suitable category of coefficients for cyclic homology, we have to repeat the definition of the cyclic vector space $A_{\#} \in \operatorname{Fun}(\Lambda, k)$, but replace the associative algebra $A$ in this definition with the tensor category $A$-bimod.

Let us explain what this means.

First, consider an arbitrary associative unital monoidal category $\mathcal{C}$ with unit object $I$ (at this point, not necessarily abelian). For any integer $n$, we have the Cartesian product $\mathcal{C}^{n}=\mathcal{C} \times \mathcal{C} \times \cdots \times \mathcal{C}$. Moreover, the product on $\mathcal{C}$ induces a product functor

$$
m: \mathcal{C}^{n} \rightarrow \mathcal{C}
$$

where if $n=0$, we let $\mathcal{C}^{n}=\mathrm{pt}$, the category with one object and one morphism, and let $m: \mathrm{pt} \rightarrow \mathcal{C}$ be the embedding of the unit object. More generally, for any finite linearly ordered set $S$ with $n$ elements, we have a product functor $m_{S}: \mathcal{C}^{S} \rightarrow \mathcal{C}$, where $\mathcal{C}^{S}=\mathcal{C}^{n}$ with multiples in the product labeled by elements of $S$. Then for any $[n],\left[n^{\prime}\right] \in \Lambda$, and any $f:\left[n^{\prime}\right] \rightarrow[n]$, 
we can define a functor $f_{!}: \mathcal{C}^{V\left(\left[n^{\prime}\right]\right)} \rightarrow \mathcal{C}^{V([n])}$ by the same formula as in (1.5):

$$
f_{!}=\prod_{v \in V([n])} m_{f^{-1}(v)}: \mathcal{C}^{V\left(\left[n^{\prime}\right]\right)}=\prod_{v \in V([n])} \mathcal{C}^{f^{-1}(v)} \rightarrow \mathcal{C}^{V([n])}
$$

The natural associativity isomorphism for the product on $\mathcal{C}$ induces natural isomorphisms $\left(f \circ f^{\prime}\right) ! \cong f_{!} \circ f_{!}^{\prime}$, and one checks easily that they satisfy natural compatibility conditions. All in all, setting $[n] \mapsto \mathcal{C}^{V([n])}, f \mapsto f$ ! defines a weak functor (a.k.a. lax functor, a.k.a. 2-functor, a.k.a. pseudofunctor in the original terminology of Grothendieck) from $\Lambda$ to the category of categories. Informally, we have a "cyclic category".

To work with weak functors, it is convenient to follow Grothendieck's approach in $\mathrm{Gr}$. Namely, instead of considering a weak functor directly, we define a category $\mathcal{C}_{\#}$ in the following way: its objects are pairs $\left\langle[n], M_{n}\right\rangle$ of an object $[n]$ of $\Lambda$ and an object $M_{n} \in \mathcal{C}^{n}$, and morphisms from $\left\langle\left[n^{\prime}\right], M_{n^{\prime}}\right\rangle$ to $\left\langle[n], M_{n}\right\rangle$ are pairs $\left\langle f, \iota_{f}\right\rangle$ of a map $f:\left[n^{\prime}\right] \rightarrow[n]$ and a bimodule map $\iota_{f}$ : $f_{!}\left(M_{n^{\prime}}\right) \rightarrow M_{n}$. A map $\left\langle f, \iota_{f}\right\rangle$ is called cocartesian if $\iota_{f}$ is an isomorphism. For the details of this construction, - in particular, for the definition of the composition of morphisms, - we refer the reader to $\mathrm{Gr}$.

The category $\mathcal{C}_{\#}$ comes equipped with a natural forgetful projection $\tau: \mathcal{C}_{\#} \rightarrow \Lambda$, and this projection is a cofibration in the sense of [Gr]. A section of this projection is a functor $\sigma: \Lambda \rightarrow \mathcal{C}_{\#}$ such that $\tau \circ \sigma=$ id (since $\Lambda$ is small, there is no harm in requiring that two functors from $\Lambda$ to itself are equal, not just isomorphic). These sections obviously form a category which we denote by $\operatorname{Sec}\left(\mathcal{C}_{\#}\right)$. Explicitly, an object $M_{\#} \in \operatorname{Sec}\left(\mathcal{C}_{\#}\right)$ is given by the following:

(i) a collection of objects $M_{n}=M_{\#}([n]) \in \mathcal{C}^{n}$, and

(ii) a collection of transition maps $\iota_{f}: f_{!} M_{n^{\prime}} \rightarrow M_{n}$ for any $n, n^{\prime}$, and $f \in \Lambda\left(\left[n^{\prime}\right],[n]\right)$,

subject to natural compatibility conditions.

A section $\sigma: \Lambda \rightarrow \mathcal{C}_{\#}$ is called cocartesian if $\sigma(f)$ is a cocartesian map for any $[n],\left[n^{\prime}\right] \in \Lambda$ and $f:\left[n^{\prime}\right] \rightarrow[n]$ - equivalently, a section is cocartesian if all the transition maps $\iota_{f}$ are isomorphisms. Cocartesian sections form a full subcategory $\operatorname{Sec}_{\text {cart }}\left(\mathcal{C}_{\#}\right)$

Lemma 2.2. The category $\operatorname{Sec}_{\text {cart }}\left(\mathcal{C}_{\#}\right)$ of cocartesian objects $M_{\#} \in \operatorname{Sec}\left(\mathcal{C}_{\#}\right)$ is equivalent to the category of the following data: 
(i) an object $M=M_{\#}([1]) \in \mathcal{C}$, and

(ii) an isomorphism $\tau: I \times M \rightarrow M \times I$ in the category $\mathcal{C}^{2}=\mathcal{C} \times \mathcal{C}$,

such that, if we denote by $\tau_{i j}$ the endomorphism of $I \times I \times M \in \mathcal{C}^{3}$ obtained by applying $\tau$ to the $i$-th and $j$-th multiple, we have $\tau_{31} \circ \tau_{12} \circ \tau_{23}=\mathrm{id}$.

Proof. Straghtforward and left to the reader.

Thus the natural forgetfull functor $\operatorname{Sec}_{\text {cart }}\left(\mathcal{C}_{\#}\right) \rightarrow \mathcal{C}, M_{\#} \mapsto M_{\#}([1])$ is faithful: an object in $\operatorname{Sec}_{\text {cart }}\left(\mathcal{C}_{\#}\right)$ is given by $M_{\#}([1])$ plus some extra structure on it, and all the higher components $M_{\#}([n]), n \geq 2$, together with the transition maps $\iota_{f}$, can be recovered from $M_{\#}([1])$ and this extra structure.

Return now to the abelian situation: we are given an associative unital algebra $A$ over a field $k$, and our monoidal category is $\mathcal{C}=A$-bimod, with the natural tensor product. Then for every $n$, the product $A$-bimod ${ }^{n}$ has a fully faithful embedding $A$-bimod ${ }^{n} \rightarrow A^{\otimes n}$-bimod, $M_{1} \times M_{2} \times \cdots \times M_{n} \mapsto$ $M_{1} \otimes M_{2} \otimes \cdots \otimes M_{n}$, and one checks easily that the multiplication functors $m_{S}$ actually extend to right-exact functors

$$
m_{S}: A^{\otimes S} \text {-bimod } \rightarrow A \text {-bimod; }
$$

for instance, one can define $m_{S}$ as

$$
m_{S}(M)=M /\left\{a_{v^{\prime}} m-m a_{v} \mid v \in S, a \in A, m \in M\right\},
$$

where $a_{v}=1 \otimes \cdots \otimes a \otimes \cdots \otimes 1 \in A^{\otimes S}$ with $a$ at the $v$-th position, and $v^{\prime} \in S$ is the next element after $v$. We can therefore define the cofibered category $A$-bimod $\# / \Lambda$ with fiber $A^{\otimes V([n])}$-bimod over $[n] \in \Lambda$, and transition functors $f_{!}$as in (2.5). We also have the category of $\operatorname{sections} \operatorname{Sec}(A$-bimod $\#)$ and the subcategory of cocartesian sections $\operatorname{Sec}_{c a r t}(A$-bimod $\#) \subset \operatorname{Sec}(A$-bimod $\#)$.

Lemma 2.3. The category $\operatorname{Sec}\left(A\right.$-bimod $\left._{\#}\right)$ is a k-linear abelian category.

Sketch of a proof. This is a general fact about cofibered categories; the proof is straightforward. The kernel $\operatorname{Ker} \phi$ and cokernel Coker $\phi$ of a map $\phi$ : $M_{\#} \rightarrow M_{\#}^{\prime}$ between objects $M_{\#}, M_{\#}^{\prime} \in \operatorname{Sec}\left(A\right.$-bimod $\left.{ }_{\#}\right)$ are taken pointwise: for every $n$, we have an exact sequence

$$
0 \rightarrow(\operatorname{Ker} \phi)([n]) \rightarrow M_{\#}([n]) \stackrel{\phi}{\rightarrow} M_{\#}^{\prime}([n]) \rightarrow(\operatorname{Coker} \phi)([n]) \rightarrow 0 .
$$

The transtition maps $\iota_{f}$ for $\operatorname{Ker} \phi$ are obtained by restriction from those for $M_{\#}$; for Coker $\phi$, one uses the fact that the functors $f_{\text {! }}$ are right-exact. 
Definition 2.4. A cyclic bimodule $M$ over a unital associative algebra $A$ is a cocartesian section $M_{\#} \in \operatorname{Sec}_{\text {cart }}\left(A\right.$-bimod $\left._{\#}\right)$. A complex of cyclic bimodules $M$. over $A$ is an object in the derived category $\mathcal{D}\left(\operatorname{Sec}\left(A\right.\right.$-bimod $\left.\left._{\#}\right)\right)$ whose homology objects are cocartsian.

Complexes of cyclic bimodules obviously form a full triangulated subcategory in $\mathcal{D}(\operatorname{Sec}(A$-bimod $\#))$; consistent notation for this category would be $\mathcal{D}_{\text {cart }}(\operatorname{Sec}(A$-bimod $\#))$, but for simplicity we will denote it $\mathcal{D} \Lambda(A$-bimod $)$. We have to define complexes separately for the following reasons:

(i) The category $\operatorname{Sec}_{c a r t}(A$-bimod $\#) \subset \operatorname{Sec}(A$-bimod $\#)$ need not be abelian - since the transition functors $f$ ! are only right-exact, the condition of being cocartesian need not be preserved when passing to kernels.

(ii) Even if $\operatorname{Sec}_{\text {cart }}(A$-bimod $\#)$ is abelian, its derived category might be much smaller than $\mathcal{D} \Lambda(A$-bimod $)$.

Example 2.5. An extreme example of (ii) is the case $A=k$ : in this case $\operatorname{Sec}(A$-bimod $\#)$ is just the category of cyclic vector spaces, $\operatorname{Fun}(\Lambda, k)$, and $E \in \operatorname{Fun}(\Lambda, k)$ is cocartesian if and only if $E(f)$ is invertible for any map $f:\left[n^{\prime}\right] \rightarrow[n]$. One deduces easily that $E$ must be a constant functor, so that $\operatorname{Sec}_{\text {cart }}(k$-bimod $\#)=k$-Vect. Then $\mathcal{D} \Lambda(k$-bimod $)$ is the full subcategory $\mathcal{D}_{\text {const }}(\Lambda, k) \subset \mathcal{D}(\Lambda, k)$ of complexes whose homology is constant. If we were to consider $\Delta^{o p p}$ instead of $\Lambda$, we would have $\mathcal{D}_{\text {const }}\left(\Delta^{o p p}, k\right) \cong \mathcal{D}(k$-Vect $)$ - since $H^{\bullet}\left(\Delta^{o p p}, k\right)=k$, the embedding $\mathcal{D}(k$-Vect $) \rightarrow \mathcal{D}\left(\Delta^{o p p}, k\right)$ is fully faithful, and $\mathcal{D}_{\text {const }}\left(\Delta^{o p p}, k\right)$ is its essential image. However, $H^{\bullet}(\Lambda, k)$ is $k[u]$, not $k$. Therefore there are maps between constant functors in $\mathcal{D}(\Lambda, k)$ which do not come from maps in $\mathcal{D}(k$-Vect), and the cones of these maps give objects in $\mathcal{D}_{\text {const }}(\Lambda, k)$ which do not come from $\mathcal{D}(k$-Vect $)$.

This phenomenon is quite common in homological algebra - examples are, for instance, the triangulated category of complexes of étale sheaves with constructible homology, the category of complex of $\mathcal{D}$-modules with holonomic homology, or the so-called "equivariant derived category" of sheaves on a topological space $X$ acted upon by a topological group $G$ (which is not in fact the derived category of anything useful). The upshot is that it is the triangulated category $\mathcal{D} \Lambda(A$-bimod $)$ which should be treated as the basic object, wherever categories are discussed.

Remark 2.6. We note one interesting property of the category $\mathcal{D}_{\text {const }}(\Lambda, k)$. Fix an integer $n \geq 1$, and consider the full subcategory $\Lambda_{\leq n} \subset \Lambda$ of objects 
$\left[n^{\prime}\right] \in \Lambda$ with $n^{\prime} \leq n$. Then one can show that $H^{\bullet}\left(\Lambda_{\leq n}, k\right)=k[u] / u^{n}$, so that we have a natural exact triangle

$$
H_{\bullet}\left(\Lambda_{\leq n}, E^{\bullet}\right) \longrightarrow H C_{\bullet}\left(E^{\bullet}\right) \stackrel{u^{n}}{\longrightarrow} H C_{\bullet+2 n}(E) \longrightarrow \text {, }
$$

for every $E^{\cdot} \in \mathcal{D}_{\text {const }}(\Lambda, k)$. We note that for any $E^{\bullet} \in \mathcal{D}(\Lambda, k)$, (1.4) extends to a spectral sequence

$$
H H_{.}\left(E^{\bullet}\right)\left[u^{-1}\right] \Rightarrow H C .(E),
$$

where the expression on the left-hand side reads as "polynomials in one formal variable $u^{-1}$ of homological degree 2 with coefficients in $H H_{\bullet}\left(E^{*}\right)$ ". Then (2.6) shows that for $E^{*} \in \mathcal{D}_{\text {const }}(\Lambda, k)$, the first $n$ differentials in (2.7) depend only on the restriction of $E^{\bullet}$ to $\Lambda_{\leq(n+1)} \subset \Lambda$. This is useful because in practice, one is often interested only in the first differential in the spectral sequence.

As in Lemma 2.2, a cyclic $A$-bimodule $M_{\#}$ essentially consists of an $A$ bimodule $M=M_{\#}([1])$ equipped with an extra structure. Explicitly, this structure is a map $\tau: A \otimes_{k} M \rightarrow M \otimes_{k} A$ which respects the $A^{\otimes 2}$-bimodule structure on both sides, and satisfies the condition $\tau_{31} \circ \tau_{12} \circ \tau_{23}=\mathrm{id}$, as in Lemma 2.2.

Another way to view this structure is the following. One checks easily that for any cyclic $A$-bimodule $M_{\#}$, the restriction $j^{*} M_{\#} \in \operatorname{Fun}\left(\Delta^{o p p}, k\right)$ is canonically isomorphic to the simplicial $k$-vector space $M_{\#}^{\Delta}$ associated to the underlying $A$-bimodule $M$ as in (2.3). By adjunction, we have a natural map

$$
\tau_{\#}: j_{!} M_{\#}^{\Delta} \rightarrow M_{\#} \cdot
$$

Then $j_{!} M_{\#}^{\Delta}$ in this formula only depends on $M \in A$-bimod, and all the structure maps which turn $M$ into the cyclic bimodule $M_{\#}$ are collected in the map $\tau_{\#}$.

We can now define cyclic homology with coefficients. The definition is rather tautological. We note that for any cyclic $A$-bimodule $M_{\#}$ - or in fact, for any $M_{\#} \in \operatorname{Sec}\left(A\right.$-bimod $\left._{\#}\right)$ - we can treat $M_{\#}$ as a cyclic vector space by forgetting the bimodule structure on its components $M_{n}$.

Definition 2.7. The cyclic homology $H C$. $\left(A, M_{\#}\right)$ with coefficients in a cyclic $A$-bimodule $M$ is equal to $H$. $\left(\Lambda, M_{\#}\right)$.

Of course, (1.4), being valid for any cyclic $k$-vector space, also applies to $H C .\left(A, M_{\#}\right)$, so that we automatically get the whole package - the Connes' 
exact sequence, the periodicity endomorphism, and the periodic cyclic homology $H P .(A, M)$. By Lemma 2.1, $H H_{\text {. }}\left(M_{\#}\right)$ coincides with $H H_{\bullet}(A, M)$ as defined in (2.2).

\section{Gauss-Manin connection.}

To illustate the usefulness of the notion of a cyclic bimodule, let us study the behavior of cyclic homology under deformations.

There are two types of deformation theory objects that one can study for an associative algebra $A$. The first is the notion of a square-zero extension of the algebra $A$ by a $A$-bimodule $M$. This is an associative algebra $\widetilde{A}$ which fits into a short exact sequence

$$
0 \longrightarrow M \stackrel{i}{\longrightarrow} \tilde{A} \stackrel{p}{\longrightarrow} A \longrightarrow 0,
$$

where $p$ is an algebra map, and $i$ is an $\widetilde{A}$-bimodule map, under the $\widetilde{A}$ bimodule structure on $M$ induced from the given $A$-bimodule structure by means of the map $p$. In other words, the multiplication on the ideal $\operatorname{Ker} p \subset$ $\widetilde{A}$ is trivial, so that the $\widetilde{A}$-bimodule structure on $\operatorname{Ker} p$ is induced by an $A$ bimodule structure, and $i$ identifies the $A$-bimodule $\operatorname{Ker} p$ with $M$. Squarezero extensions are classified up to an isomorphism by elements in the second Hochschild cohomology group $H H^{2}(A, M)$, defined as

$$
H H^{\bullet}(A, M)=\operatorname{Ext}_{A^{\text {opp }} \otimes A}(A, M) .
$$

In this setting, we can consider the cyclic homology of the algebra $\widetilde{A}$ and compare with the cyclic homology of $A$. Th. Goodwillie's theorem [Go claims that if the base field $k$ has characteristic 0 , the natural map

$$
H P .(\widetilde{A}) \rightarrow H P .(A)
$$

is an isomorphism, and there is also some information on the behaviour of $H C$. $(A)$.

A second type of deformation theory data includes a commutative $k$ algebra $R$ with a maximal ideal $\mathfrak{m} \subset R$. A deformation $A_{R}$ of the algebra $A$ over $R$ is a flat associative unital algebra $A_{R}$ over $R$ equipped with an isomorphism $A_{R} / \mathfrak{m} \cong A$. In this case, one can form the relative cyclic $R$ module $A_{R \#}$ by taking the tensor products over $R$; thus we have relative homology $H H_{\text {. }}\left(A_{R} / R\right), H C .\left(A_{R} / R\right), H P .\left(A_{R} / R\right)$. The fundamental fact discovered by E. Getzler $\mathrm{Ge}$ is that we have an analog of the Gauss-Manin 
connection: if Spec $R$ is smooth, the $R$-module $H P_{i}\left(A_{R} / R\right)$ carries a canonical flat connection for every $i$.

Consider now the case when $R$ is not smooth but, on the contrary, local Artin. Moreover, assume that $\mathfrak{m}^{2}=0$, so that $R$ is itself a (commutative) square-zero extension of $k$. Then a deformation $A_{R}$ of $A$ over $R$ is also a square-zero extension of $A$, by the bimodule $A \otimes \mathfrak{m}(\mathfrak{m}$ here is taken as a $k$-vector space). But this square-zero extension is special - for a general square-zero extension $\widetilde{A}$ of $A$ by some $M \in A$-bimod, there does not exist any analog of the relative cyclic $R$-module $A_{R \#} \in \operatorname{Fun}(\Lambda, R)$.

We observe the following: the data needed to define such an analog is precisely a cyclic bimodule structure on the bimodule $M$.

Namely, assume given a square-zero extension $\widetilde{A}$ of the algebra $A$ by some $A$-bimodule $M$, and consider the cyclic $k$-vector space $\widetilde{A}_{\#} \in \operatorname{Fun}(\Lambda, k)$. Let us equip $\widetilde{A}$ with a descreasing two-step filtration $F^{*}$ by setting $F^{1} \widetilde{A}=M$. Then this induces a decreasing filtration $F^{\bullet}$ on tensor powers $\widetilde{A}^{\otimes n}$. Since $\widetilde{A}$ is square-zero, $F^{\bullet}$ is compatible with the multiplication maps; therefore we also have a filtration $F^{\bullet}$ on $\widetilde{A}_{\#}$. Consider the quotient

$$
\overline{A_{\#}}=\widetilde{A}_{\#} / F^{2} \widetilde{A}_{\#}
$$

One checks easily that $\operatorname{gr}_{F}^{0} \widetilde{A}_{\#} \cong A_{\#}$ and $\operatorname{gr}_{F}^{1} \widetilde{A}_{\#} \cong j_{!} M_{\#}^{\Delta}$ in a canonical way, so that $\overline{A_{\#}}$ fits into a canonical short exact sequence

$$
0 \longrightarrow j_{!} M_{\#}^{\Delta} \longrightarrow \overline{A_{\#}} \longrightarrow A_{\#} \longrightarrow 0
$$

of cyclic $k$-vector spaces.

Now assume in addition that $M$ is equipped with a structure of a cyclic $A$-bimodule $M_{\#}$, so that $M_{\#}^{\Delta} \cong j^{*} M_{\#}$, and we have the structure map $\tau_{\#}: j_{!} M_{\#}^{\Delta} \rightarrow M_{\#}$. Then we can compose the extension (3.1) with the map $\tau_{\#}$, to obtain a commutative diagram

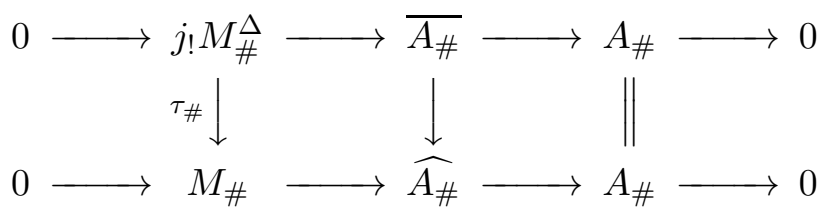

of short exact sequences in $\operatorname{Fun}(\Lambda, k)$, with cartesian left square. It is easy to check that when $\widetilde{A}=A_{R}$ for some square-zero $R$, so that $M=A \otimes \mathfrak{m}$, and we take the cyclic $A$-bimodule structure on $M$ induced by the tautological structure on $A$, then $\widehat{A_{\#}}$ coincides precisely with the relative cyclic 
object $A_{R \#}$ (which we consider as a $k$-vector space, forgetting the $R$-module structure).

We believe that this is the proper generality for the Getzler connection; in this setting, the main result reads as follows.

Proposition 3.1. Assume given a square-zero extension $\widetilde{A}$ of an associative algebra $A$ by an A-bimodule $M$, and assume that $M$ is equipped with a structure of a cyclic A-bimodule. Then the long exact sequence

$$
H P_{.}(A, M) \longrightarrow H_{.}\left(\widehat{A_{\#}}\right) \longrightarrow H_{\bullet}(A) \longrightarrow
$$

of periodic cyclic homology induced by the second row in (3.2) admits a canonical splitting $H P .(A) \rightarrow H P .\left(\widehat{A_{\#}}\right)$.

Proof. By definition, we have two natural maps

$$
\begin{aligned}
& H P .\left(\overline{A_{\#}}\right) \rightarrow H P .\left(A_{\#}\right)=H P .(A), \\
& H P .\left(\overline{A_{\#}}\right) \rightarrow H P .\left(\widehat{A_{\#}}\right),
\end{aligned}
$$

and the cone of the first map is isomorphic to $H P .\left(j_{!} M_{\#}^{\Delta}\right)$. Since $j$ ! is exact, we have $H C \cdot\left(j_{!} M_{\#}^{\Delta}\right) \cong H H_{0}\left(M_{\#}\right)$, and the periodicity map $u$ : $H C .\left(j_{!} M_{\#}^{\Delta}\right) \rightarrow H C_{.-2}\left(j_{!} M_{\#}^{\Delta}\right)$ is equal to 0 , so that $H P .\left(j_{!} M_{\#}^{\Delta}\right)=0$. Thus the first map in (3.3) is an isomorphism, and the second map is then the required splitting.

Corollary 3.2. Assume given a commutative $k$-algebra $R$ with a maximal ideal $\mathfrak{m} \subset R$, and a deformation $A_{R}$ of the algebra $A$ over $R$. Then if $\operatorname{Spec} R$ is smooth, the $R$-modules $H P .\left(A_{R} / R\right)$ carry a natural connection.

Sketch of a proof. Consider the $R \otimes R$-algebras $A_{R} \otimes R$ and $R \otimes A_{R}$, and their restrictions to the first infinitesemal neighborhood of the diagonal in $\operatorname{Spec}(R \otimes R)=\operatorname{Spec} R \times \operatorname{Spec} R$. Then Proposition 3.1, suitably generalized, shows that $H P .(-)$ of these two restrictions are canonically isomorphic. It is well-known that giving such an isomorphism is equivalent to giving a connection on $H P .\left(A_{R} / R\right)$.

We note that we do not claim that the connection is flat. It certainly is, at least in characteristic 0; but our present method does not allow one to go beyond square-zero extensions. Thus we cannot analyse the second infinitesemal neighborhood of the diagonal in $\operatorname{Spec}(R \otimes R)$, and we cannot prove flatness. 
Unfortunately, at present, we do not understand what is the proper cyclic bimodule context for higher-level infinitesemal extensions. Of course, if one is only interested in an $R$-deformation $\widetilde{A}=A_{R}$ over an Artin local base $R$, not in its cyclic bimodule generalizations, one can use Goodwillie's Theorem: using the full cyclic object $\widetilde{A}_{\#}$ instead of its quotient $\overline{A_{\#}}$ in Proposition 3.1 immediately gives a splitting $H P .(A) \rightarrow H P .\left(A_{R} / R\right)$ of the augmentation map $H P .\left(A_{R} / R\right) \rightarrow H P$. $(A)$, and this extends by $R$-linearity to an isomorphism $H P_{.}\left(A_{R} / R\right) \cong H P .(A) \otimes R$. However, this is not quite satisfactory from the conceptual point of view, and it does not work in positive characteristic (where Goodwillie's Theorem is simply not true). If char $k \neq 2$, the latter can be cured by using $\widetilde{A}_{\#} / F^{3} \widetilde{A}_{\#}$, but the former remains. We plan to return to this elsewhere.

\section{Categorical approach.}

Let us now try to define cyclic homology in a more general setting - we will attempt to replace $A$-bimod with an arbitrary associative unital $k$-linear tensor category $\mathcal{C}$ with a unit object $I \in \mathcal{C}$. We do not assume that $\mathcal{C}$ is symmetric in any way. However, we will assume that the tensor product $-\otimes-$ is right-exact in each variable, and we will need to impose additional technical assumptions later on.

The first thing to do is to try to define Hochschild homology; so, let us look more closely at (2.1). The formula in the right-hand side looks symmetric, but this is an optical illusion - the two copies of $A$ are completely different objects: one is a left module over $A^{o p p} \otimes A$, and the other is a right module ( $A$ just happens to have both structures at the same time). It is better to separate them and introduce the functor

$$
\operatorname{tr}: A \text {-bimod } \rightarrow k \text {-Vect }
$$

by $\operatorname{tr}(M)=M \otimes_{A^{o p p} \otimes A} A-$ or, equivalently, by

$$
\operatorname{tr}(M)=M /\{a m-m a \mid a \in A, m \in M\} .
$$

Then $\operatorname{tr}$ is a right-exact functor, and we have $H H_{\bullet}(A, M)=L^{\bullet} \operatorname{tr}(M)$.

We want to emphasize that the functor $\operatorname{tr}$ can not be recovered from the tensor structure on $A$-bimod - this really is an extra piece of data. For a general tensor category $\mathcal{C}$, it does not exist a priori; we have to impose it as an additional structure.

Let us axiomatize the situation. First, forget for the moment about the $k$-linear and abelian structure on $\mathcal{C}$ - let us treat it simply as a monoidal category. Assume given some other category $\mathcal{B}$ and a functor $T: \mathcal{C} \rightarrow \mathcal{B}$. 
Definition 4.1. The functor $T: \mathcal{C} \rightarrow \mathcal{B}$ is a trace functor if it is extended to a functor $\mathcal{C}_{\#} \rightarrow \mathcal{B}$ which sends any cocartesian map $f: M \rightarrow M^{\prime}$ in $\mathcal{C}_{\#}$ to an invertible map.

Another way to say the same thing is the following: the categories $\operatorname{Fun}\left(\mathcal{C}^{n}, \mathcal{B}\right)$ of functors from $\mathcal{C}^{n}$ to $\mathcal{B}$ form a fibered category over $\Lambda$, and a trace functor is a cartesian section of this fibration. Explicitly, a trace functor is defined by $T: \mathcal{C} \rightarrow \mathcal{B}$ and a collection of isomorphisms

$$
T\left(M \otimes M^{\prime}\right) \rightarrow T\left(M^{\prime} \otimes M\right)
$$

for any $M, M^{\prime} \in \mathcal{C}$ which are functorial in $M$ and $M^{\prime}$ and satisfy some compatibility conditions analogous to those in Lemma 2.2, we leave it to the reader to write down these conditions precisely. Thus $T$ has a trace-like property with respect to the product in $\mathcal{C}$, and this motivates our terminology.

Recall now that $\mathcal{C}$ is a $k$-linear abelian category. To define Hochschild homology, we have to assume that it is equipped with a right-exact trace functor $\operatorname{tr}: \mathcal{C} \rightarrow k$-Vect; then for any $M \in \mathcal{C}$, we set

$$
H H_{.}(M)=L^{\bullet} \operatorname{tr}(M) \text {. }
$$

Lemma 4.2. The functor tr : A-bimod $\rightarrow k$-Vect canonically extends to a right-exact trace functor in the sense of Definition 4.1 .

Proof. For any object $\left\langle[n], M_{n}\right\rangle \in A$-bimod $_{\#},[n] \in \Lambda, M_{n} \in A^{\otimes n}$-bimod, let

$$
\operatorname{tr}\left(\left\langle[n], M_{n}\right\rangle\right)=M_{n} /\left\{a_{v^{\prime}} m-m a_{v} \mid v \in V([n]), m \in M_{n}, a \in A\right\},
$$

where $a_{v}=1 \otimes 1 \otimes \cdots \otimes a \otimes \cdots \otimes 1 \in A^{\otimes V([n])}$ has $a$ in the multiple corresponding to $v \in V([n])$, and $v^{\prime} \in V([n])$ is the next marked point after $v$ counting clockwise. The compatibility with maps in the category $A$-bimod $\#$ is obvious.

We note that here, in the case $\mathcal{C}=A$-bimod, the category $A$-bimod $\#$ is actually larger than what we would have had purely from the monoidal structure on $\mathcal{C}: M_{n}$ is allowed to be an arbitrary $A^{\otimes n}$-bimodule, not a collection of $n A$-bimodules. To do the same for general $k$-linear $\mathcal{C}$, we need to replace $A^{\otimes n}$-bimod with some version of the tensor product $\mathcal{C}^{\otimes n}$. Here we have a difficulty: for various technical reasons, it is not clear how to define tensors products for sufficiently general abelian categories.

One way around it is the following. For any (small) $k$-linear abelian category $\mathcal{B}$, a $k$-linear functor $\mathcal{B}^{o p p} \rightarrow k$-Vect is left-exact if and only if it 
is a sheaf for for the canonical Grothendieck topology on $\mathcal{B}([\mathrm{BD}, 5, \S 10])$; the category $\operatorname{Shv}(\mathcal{B})$ of such functors is abelian and $k$-linear, and $\mathcal{B}$ itself is naturally embedded into $\operatorname{Shv}(\mathcal{B})$ by Yoneda. The embedding is a fully faithfull exact functor. Every functor in $\operatorname{Shv}(\mathcal{B})$ is in fact a direct limit of representable functors, so that $\operatorname{Shv}(\mathcal{B})$ is an inductive completion of the abelian category $\mathcal{B}$. Now, if are given two (small) $k$-linear abelian categories $\mathcal{B}_{1}, \mathcal{B}_{2}$, then their product $\mathcal{B}_{1} \times \mathcal{B}_{2}$ is no longer abelian. However, we still have the abelian category $\operatorname{Shv}\left(\mathcal{B}_{1} \times \mathcal{B}_{2}\right)$ of bilinear functors $\mathcal{B}_{1}^{\text {opp }} \times \mathcal{B}_{2}^{\text {opp }} \rightarrow$ $k$-Vect which are left-exact in each variable, and the same goes for polylinear functors.

Moreover, for any right-exact functor $F: \mathcal{B}_{1} \rightarrow \mathcal{B}_{2}$ between small abelian categories, we have the restriction functor $F^{*}: \operatorname{Shv}\left(\mathcal{B}_{2}\right) \rightarrow \operatorname{Shv}\left(\mathcal{B}_{1}\right)$, which is left-exact, and its left-adjoint $F_{!}: \operatorname{Shv}\left(\mathcal{B}_{1}\right) \rightarrow \operatorname{Shv}\left(\mathcal{B}_{2}\right)$, which is rightexact. The functor $F_{\text {! }}$ is an extension of the functor $F$ : on Yoneda images $\mathcal{B}_{i} \subset \operatorname{Shv}\left(\mathcal{B}_{i}\right)$, we have $F !=F$. And, again, the same works for polylinear functors.

In particular, given our $k$-linear abelian tensor category $\mathcal{C}$, we can form the category $\operatorname{Shv}(\mathcal{C})_{\#}$ of pairs $\langle E,[n]\rangle,[n] \in \Lambda, E \in \operatorname{Shv}\left(\mathcal{C}^{n}\right)$, with a map from $\left\langle E^{\prime},\left[n^{\prime}\right]\right\rangle$ to $\langle E,[n]\rangle$ given by a pair of a map $f:\left[n^{\prime}\right] \rightarrow[n]$ and either a map $E^{\prime} \rightarrow\left(f_{!}\right)^{*} E$, or map $\left(f_{!}\right) ! E^{\prime} \rightarrow E$ - this is equivalent by adjunction. Then $\operatorname{Shv}(\mathcal{C})_{\#}$ is bifibered category over $\Lambda$ in the sense of $\mathrm{Gr}$.

The category of sections $\Lambda \rightarrow \operatorname{Shv}(\mathcal{C})_{\#}$ of this bifibration can also be described as the full subcategory $\operatorname{Shv}\left(\mathcal{C}_{\#}\right) \subset \operatorname{Fun}\left(\mathcal{C}_{\#}^{o p p}, k\right)$ spanned by those functors $E_{\#}: \mathcal{C}_{\#}^{o p p} \rightarrow k$-Vect whose restriction to $\left(\mathcal{C}^{o p p}\right)^{n} \subset \mathcal{C}_{\#}^{o p p}$ is a sheaf that is, an object in $\operatorname{Shv}\left(\mathcal{C}^{n}\right) \subset \operatorname{Fun}\left(\left(\mathcal{C}^{o p p}\right)^{n}, k\right)$. Since the transition functors $\left(f_{!}\right)$! are right-exact, $\operatorname{Shv}\left(\mathcal{C}_{\#}\right)$ is an abelian category (this is proved in exactly the same way as Lemma 2.3).

We denote by $\operatorname{Shv}_{\text {cart }}\left(\mathcal{C}_{\#}\right) \subset \operatorname{Shv}\left(\mathcal{C}_{\#}\right)$ the full subcategory of sections $E$ : $\Lambda \rightarrow \operatorname{Shv}(\mathcal{C})_{\#}$ which are cocartesian, and moreover, are such that $E([1]) \in$ $\operatorname{Shv}(\mathcal{C})$ actually lies in the Yoneda image $\mathcal{C} \subset \operatorname{Shv}(\mathcal{C})$. We also denote by $\mathcal{D} \Lambda(\mathcal{C}) \subset \mathcal{D}\left(\operatorname{Shv}\left(\mathcal{C}_{\#}\right)\right)$ the full triangulated subcategory of complexes $E_{\#}^{\bullet} \in \mathcal{D}\left(\operatorname{Shv}\left(\mathcal{C}_{\#}\right)\right)$ with homology in $\operatorname{Shv}_{\text {cart }}\left(\mathcal{C}_{\#}\right)$.

If $\mathcal{C}$ is the category of $A$-bimodules for some algebra $A$-or better yet, of $A$-bimodules of cardinality not more than that of $A \times \mathbb{N}$, so that $\mathcal{C}$ is small - then $\operatorname{Shv}(\mathcal{C})$ is equivalent to $A$-bimod (one shows easily that every sheaf $E \in \operatorname{Shv}(\mathcal{C})$ is completely determined by its value at $\left.A^{o p p} \otimes A \in \mathcal{C}\right)$. In this case, $\mathcal{D} \Lambda(\mathcal{C})$ is our old category $\mathcal{D} \Lambda(A$-bimod $)$.

Now, we assume that $\mathcal{C}$ is equipped with a right-exact trace functor $\operatorname{tr}: \mathcal{C} \rightarrow k$-Vect, we would like to define cyclic homology $H C .\left(M_{\bullet}\right)$ for any 
$M_{.} \in \mathcal{D} \Lambda(\mathcal{C})$, and we immediately notice a problem: for a general $\mathcal{C}$, we do not have a forgetful functor to vector spaces. However, it turns out that the forgetful functor is not needed for the definition - it can be replaced with the trace functor $\mathrm{tr}$.

We proceed as follows. By definition, tr is extended to a functor $\mathcal{C}_{\#} \rightarrow$ $k$-Vect; we extend it canonically to a functor $\operatorname{Shv}(\mathcal{C})_{\#} \rightarrow k$-Vect, and consider the product

$$
\operatorname{tr} \times \tau: \operatorname{Shv}(\mathcal{C})_{\#} \rightarrow k-\operatorname{Vect} \times \Lambda,
$$

where $\tau: \operatorname{Shv}(\mathcal{C})_{\#} \rightarrow \Lambda$ is the projection. This is a functor compatible with the projections to $\Lambda$, and therefore, it induces a functor of the categories of sections. The category of sections of the projection $k$-Vect $\times \Lambda \rightarrow \Lambda$ is tautologically the same as $\operatorname{Fun}(\Lambda, k$-Vect $)$, so that we have a functor

$$
\operatorname{tr}_{\#}: \operatorname{Shv}\left(\mathcal{C}_{\#}\right) \rightarrow \operatorname{Fun}(\Lambda, k) .
$$

One checks easily that this functor is right-exact.

Definition 4.3. For any $M_{\#} \in \operatorname{Sec}\left(\mathcal{C}_{\#}\right)$, its cyclic homology $H C$. $\left(M_{\#}\right)$ is defined by

$$
H C .\left(M_{\#}\right) \stackrel{\text { def }}{=} H C \cdot\left(L^{\bullet} \operatorname{tr}_{\#}\left(M_{\#}\right)\right)=H_{\bullet}\left(\Lambda, L^{\bullet} \operatorname{tr}_{\#}\left(M_{\#}\right)\right) .
$$

Definition 4.4. The pair $\langle\mathcal{C}, \operatorname{tr}\rangle$ is called homologically clean if for any $n$, the category $\operatorname{Shv}\left(\mathcal{C}^{n}\right)$ has enough objects $E$ such that

(i) $E$ is acyclic both for functors $\left(f_{!}\right) !: \operatorname{Shv}\left(\mathcal{C}^{n}\right) \rightarrow \operatorname{Shv}\left(\mathcal{C}^{n^{\prime}}\right)$, for any $f:[n] \rightarrow\left[n^{\prime}\right]$, and for the trace functor $\operatorname{tr}: \operatorname{Shv}\left(\mathcal{C}^{n}\right) \rightarrow k$-Vect, and

(ii) for any $f:[n] \rightarrow\left[n^{\prime}\right],\left(f_{!}\right) ! E \in \operatorname{Shv}\left(\mathcal{C}^{n^{\prime}}\right)$ is acyclic for $\operatorname{tr}: \operatorname{Shv}\left(\mathcal{C}^{n^{\prime}}\right) \rightarrow$ $k$-Vect.

Example 4.5. Assume that the category $\mathcal{C}$ has enough projectives, and moreover, $P_{1} \otimes P_{2}$ is projective for any projective $P_{1}, P_{2} \in \mathcal{C}$ (this is satisfied, for instance, for $\mathcal{C}=A$-bimod). Then the pair $\langle\mathcal{C}, \operatorname{tr}\rangle$ is homologically clean, for any trace functor $\operatorname{tr}$. Indeed, $\operatorname{Shv}\left(\mathcal{C}^{n}\right)$ then also has enough projectives, say sums of objects

$$
P=P_{1} \otimes P_{2} \otimes \cdots \otimes P_{n} \in \operatorname{Shv}\left(\mathcal{C}^{n}\right)
$$

for projective $P_{1}, \ldots, P_{n} \in \mathcal{C} \subset \operatorname{Shv}(\mathcal{C})$, and these projectives automatically satisfy the condition (i). To check (ii), one decomposes $f:[n] \rightarrow\left[n^{\prime}\right]$ into a surjection $p:[n] \rightarrow\left[n^{\prime \prime}\right]$ and an injection $i:\left[n^{\prime \prime}\right] \rightarrow\left[n^{\prime}\right]$. Since the tensor 
product of projective objects is projective, $\left(p_{!}\right)_{!}(P) \in \operatorname{Shv}\left(\mathcal{C}^{n^{\prime \prime}}\right)$ is also an object of the type (4.3), so we may as well assume that $f$ is injective. Then one can find a left-inverse map $f^{\prime}:\left[n^{\prime}\right] \rightarrow[n], f^{\prime} \circ f=$ id; since $P^{\prime}=\left(f_{!}\right)_{!}(P)$ is obviously acyclic for $\left(f_{!}^{\prime}\right)_{!}$, and $\left(f_{!}^{\prime}\right)_{!}\left(P^{\prime}\right)=\left(\left(f^{\prime} \circ f\right)_{!}\right)_{!}(P)=P$ is acyclic for $\operatorname{tr}, P^{\prime}$ itself is acyclic for $\operatorname{tr}=\operatorname{tr} \circ\left(f_{!}^{\prime}\right)$ !

Lemma 4.6. Assume that $\langle\mathcal{C}, \operatorname{tr}\rangle$ is homologically clean. Then for any object $[n] \in \Lambda$ and any $M_{\#} \in \operatorname{Shv}\left(\mathcal{C}_{\#}\right)$, we have

$$
L^{\cdot} \operatorname{tr}_{\#}\left(M_{\#}\right)([n]) \cong L^{\cdot} \operatorname{tr}\left(M_{\#}([n])\right) .
$$

For any $M_{\#}^{\dot{*}} \in \mathcal{D} \Lambda(\mathcal{C})$, we have $L^{\bullet} \operatorname{tr}_{\#}\left(M_{\#}\right) \in \mathcal{D}_{\text {const }}(\Lambda, k) \subset \mathcal{D}(\Lambda, k)$.

Proof. The natural restriction functor $\operatorname{Shv}\left(\mathcal{C}_{\#}\right) \rightarrow \operatorname{Shv}\left(\mathcal{C}^{n}\right), M_{\#} \mapsto M_{\#}([m])$ has a left-adjoint functor $I_{n !}: \operatorname{Shv}\left(\mathcal{C}^{n}\right) \rightarrow \operatorname{Shv}\left(\mathcal{C}_{\#}\right)$; explicitly, it is given by

$$
I_{n !}(E)\left(\left[n^{\prime}\right]\right)=\bigoplus_{f:[n] \rightarrow\left[n^{\prime}\right]}\left(f_{!}\right) !(E) .
$$

Let us say that an object $E \in \operatorname{Shv}\left(\mathcal{C}^{n}\right)$ is admissible if it satisfies the conditions (i), (ii) of Definition 4.4. By assumption, $\operatorname{Shv}\left(\mathcal{C}^{n}\right)$ has enough admissible objects for any $n$. Then $\operatorname{Shv}\left(\mathcal{C}_{\#}\right)$ has enough objects of the form $I_{n !} E$, $[n] \in \Lambda, E \in \operatorname{Shv}\left(\mathcal{C}^{n}\right)$ admissible, and to prove the first claim, it suffices to consider $M_{\#}=I_{n !} E$ of this form. In degree 0 , (4.4) is the definition of the functor $\operatorname{tr}_{\#}$, and the higher degree terms in the right-hand side vanish by Definition 4.4 (ii). Therefore it suffices to prove that $M_{\#}=I_{n !} E$ is acyclic for the functor $\operatorname{tr}_{\#}$. This is obvious: applying $\operatorname{tr}_{\#}$ to any short exact sequence

$$
0 \longrightarrow M_{\#}^{\prime} \longrightarrow M_{\#}^{\prime \prime} \longrightarrow M_{\#} \longrightarrow 0
$$

in $\operatorname{Shv}\left(\mathcal{C}_{\#}\right)$, we see that, since $M_{\#}\left(\left[n^{\prime}\right]\right)$ is acyclic for any $\left[n^{\prime}\right] \in \Lambda$, the sequence

$$
0 \longrightarrow \operatorname{tr} M_{\#}^{\prime}\left(\left[n^{\prime}\right]\right) \longrightarrow \operatorname{tr} M_{\#}^{\prime \prime}\left(\left[n^{\prime}\right]\right) \longrightarrow \operatorname{tr} M_{\#}\left(\left[n^{\prime}\right]\right) \longrightarrow 0
$$

is exact; this means that

$$
0 \longrightarrow \operatorname{tr} M_{\#}^{\prime} \longrightarrow \operatorname{tr} M_{\#}^{\prime \prime} \longrightarrow \operatorname{tr} M_{\#} \longrightarrow 0
$$

is an exact sequence in $\operatorname{Fun}(\Lambda, k)$, and this means that $M_{\#}$ is indeed acyclic for $\operatorname{tr}_{\#}$.

With the first claim proved, the second amounts to showing that the natural map

$$
L^{\bullet} \operatorname{tr} \circ L^{\bullet}\left(f_{!}\right) !(E) \rightarrow L^{\bullet} \operatorname{tr}(E)
$$


is a quasiismorphism for any $f:[n] \rightarrow\left[n^{\prime}\right]$ and any $E \in \operatorname{Shv}\left(\mathcal{C}^{n}\right)$. It suffices to prove it for admissible $M$; then the higher derived functors vanish, and the isomorphism $\operatorname{tr} \circ\left(f_{!}\right) !$! $\operatorname{tr}$ is Definition 4.1 ,

Lemma 4.7. In the assumptions of Lemma 4.6, for any complex $M_{\#}^{\bullet} \in$ $\mathcal{D} \Lambda(\mathcal{C})$ with the first component $M^{\bullet}=M_{\#}^{\bullet}([1])$ we have

$$
H_{.}\left(M^{\bullet}\right) \cong H_{\bullet}\left(L^{\bullet} \operatorname{tr}_{\#}\left(M_{\#}^{\bullet}\right)\right) \text {. }
$$

Proof. By Lemma 4.6, the left-hand side, $H H_{.}\left(M^{\bullet}\right)$, is canonically isomorphic to the complex $L^{\bullet} \operatorname{tr}_{\#}\left(M_{\#}^{\bullet}\right) \in \mathcal{D}(\Lambda, k)$ evaluated at $[1] \in \Lambda$, and moreover, $L^{\cdot} \operatorname{tr}_{\#}\left(M_{\#}^{*}\right)$ lies in the subcategory $\mathcal{D}_{\text {const }}(\Lambda, k) \subset \mathcal{D}(\Lambda, k)$. It remains to apply the general fact: for any $E^{*} \in \mathcal{D}_{\text {const }}(\Lambda, k)$, we have a natural isomorphism $H H_{\bullet}\left(E^{\bullet}\right) \cong E^{\bullet}([1])$. Indeed, by definition we have

$$
H_{\bullet}\left(E^{\bullet}\right)=H_{\bullet}\left(\Delta^{o p p}, j^{*} E^{\bullet}\right),
$$

and $j^{*} E^{\bullet}$ lies in the category $\mathcal{D}_{\text {const }}\left(\Delta^{o p p}, k\right)$ which is equivalent to $\mathcal{D}(k-V e c t)$ (see Example 2.5, and also Remark 2.6, the isomorphism we constructed here is a special case of (2.6) for $n=1$ ).

The Lemma shows that if the pair $\langle\mathcal{C}, \operatorname{tr}\rangle$ is homologically clean, Definition 4.3 is consistent with (4.2), and we get the whole periodicity package of (1.4) - the periodicity map $u$, the Connes' exact sequence

$$
H H_{\bullet}\left(M^{\bullet}\right) \longrightarrow H C .\left(M^{\bullet}\right) \stackrel{u}{\longrightarrow} H C_{\cdot-2}\left(M^{\bullet}\right) \longrightarrow,
$$

and the periodic cyclic homology $H P .\left(M^{\bullet}\right)$.

In general, objects in $\mathcal{D} \Lambda(\mathcal{C})$ may be hard to construct, but we always have at least one - the identity section $\mathrm{I}_{\#}: \Lambda \rightarrow \operatorname{Shv}(\mathcal{C})_{\#}$, given by

$$
\mathrm{I}_{\#}([n])=\mathrm{I}^{\otimes n} \in \mathcal{C}^{\otimes n},
$$

where $\mathrm{I} \in \mathcal{C}$ is the unit object. Thus we can define cyclic homology of a tensor category equipped with a trace functor.

Definition 4.8. For any $k$-linear abelian unital tensor category $\mathcal{C}$ equipped with a trace functor $\operatorname{tr}: \mathcal{C} \rightarrow k$-Vect, its Hochschild and cyclic homology is given by

$$
H H_{\bullet}(\mathcal{C}, \text { tr }) \stackrel{\text { def }}{=} H H_{\bullet}(\mathrm{I}), \quad H C .(\mathcal{C}, \operatorname{tr}) \stackrel{\text { def }}{=} H C .\left(\mathrm{I}_{\#}\right),
$$

where $\mathrm{I} \in \mathcal{C}$ is the unit object, and $\mathrm{I}_{\#} \in \mathcal{D} \Lambda(\mathcal{C})$ is the identity section. 
We now have to check that in the case $\mathcal{C}=A$-bimod, Definition 4.3 is compatible with our earlier Definition 2.7 - in other words, that the cyclic homology computed by means of the forgetfull functor is the same as the cyclic homology computed by means of the trace. This is not at all trivial. Indeed, if for instance $M_{\#} \in \operatorname{Shv}\left(\mathcal{C}_{\#}\right)$ is cocartesian, then, while $L^{\bullet} \operatorname{tr}^{\#} M_{\#}$ lies in the subcategory $\mathcal{D}_{\text {const }}(\Lambda, k) \subset \mathcal{D}(\Lambda, k)$, the same is certainly not true for the object $M_{\#} \in \operatorname{Fun}(\Lambda, k)$ obtained by forgetting the bimodule structure on $M_{n}$.

Thus these two objects are different. However, they do become equal after taking cyclic (or Hochschild, or periodic cyclic) homology. Namely, for any $M_{\#} \in \operatorname{Sec}(A$-bimod $\#)$ we have a natural map

$$
M_{\#} \rightarrow L^{\cdot} \operatorname{tr}^{\#} M_{\#}
$$

in the derived category $\mathcal{D}(\Lambda, k)$, and we have the following result.

Proposition 4.9. For every $M_{\#} \in \operatorname{Sec}\left(A-\operatorname{bimod}_{\#}\right)$, the natural map (4.6) induces isomorphisms

$$
\begin{gathered}
H H_{.}\left(M_{\#}\right) \cong H H_{.}\left(L^{\bullet} \operatorname{tr} M_{\#}\right), \\
H C .\left(M_{\#}\right) \cong H C .\left(L^{\cdot} \operatorname{tr} M_{\#}\right), \\
H P .\left(M_{\#}\right) \cong H P .\left(L^{\bullet} \operatorname{tr} M_{\#}\right) .
\end{gathered}
$$

Proof. By (1.4), it suffices to consider HC.(-); as in the proof of Lemma4.6, it suffices to consider $M_{\#}=I_{n !} E$ given in (4.5), with $E$ being the free bimodule

$$
E=\left(A^{\text {opp }} \otimes A\right)^{\otimes n} \in \operatorname{Shv}\left(\mathcal{C}^{n}\right)=A^{\otimes n} \text {-bimod }
$$

for some fixed $n$. Explicitly, we have

$$
I_{n !} E\left(\left[n^{\prime}\right]\right)=\bigoplus_{f:[n] \rightarrow\left[n^{\prime}\right]} \bigotimes_{v^{\prime} \in V\left(\left[n^{\prime}\right]\right)} A^{\text {opp }} \otimes A^{\otimes f^{-1}\left(v^{\prime}\right)}
$$

for any $\left[n^{\prime}\right] \in \Lambda$. Then $L^{p} \operatorname{tr}_{\#} I_{n !} E=0$ for $p \geq 1$, and one checks easily that

$$
\operatorname{tr}_{\#} I_{n !} E=i_{n !} \operatorname{tr} E=i_{n !} A^{\otimes n} \in \operatorname{Fun}(\Lambda, k),
$$

where $i_{n}: \mathrm{pt} \rightarrow \Lambda$ is the embedding of the object $[n] \in \Lambda$ (pt is the category with one object and one morphism). Therefore

$$
H C_{0}\left(L^{\bullet} \operatorname{tr}_{\#} I_{n !} E\right)=H_{\bullet}\left(\Lambda, i_{n !} A^{\otimes n}\right)=A^{\otimes n},
$$

and $H C_{p}\left(L^{\bullet} \operatorname{tr}_{\#} i_{n !} E\right)=0$ for $p \geq 1$. We have to compare it with $H C .\left(i_{n !} E\right)$. 
To do this, consider the category $\Lambda_{[n]}$ of objects $\left[n^{\prime}\right] \in \Lambda$ equipped with a map $[n] \rightarrow\left[n^{\prime}\right]$, and let $j_{n}: \Lambda_{[n]} \rightarrow \Lambda$ be the forgetful functor. Then $j_{n}$ is obviously a discrete cofibration. Comparing (1.2) and (4.7), we see that

$$
I_{n !} E=j_{n !} E_{\#}^{[n]}
$$

for some $E_{\#}^{[n]} \in \operatorname{Fun}\left(\Lambda_{[n]}\right)$. Moreover, fix once and for all a map [1] $\rightarrow[n]$. Then we see that the discrete cofibration $j_{n}: \Lambda_{[n]} \rightarrow \Lambda$ factors through the discrete cofibration $j: \Lambda_{[1]}=\Delta^{o p p} \rightarrow \Lambda$ by means of a discrete cobifbration $\gamma_{n}: \Lambda_{[n]} \rightarrow \Lambda_{[1]}$, and we observe that

$$
E_{\#}^{[n]}\left(\left[n^{\prime}\right]\right)=\left(A^{o p p}\right)^{\otimes n^{\prime}} \otimes A^{\otimes n}
$$

only depends on $\gamma_{n}\left(\left[n^{\prime}\right]\right) \in \Delta^{o p p}$. More precisely, we have $E_{\#}^{[n]}=\gamma_{n}^{*} E_{n}^{\Delta}$, where $E_{n}^{\Delta} \in \operatorname{Fun}\left(\Delta^{o p p}, k\right)$ is as in (2.3) , and $E_{n}$ is the free $A$-bimodule

$$
E_{n}=A^{o p p} \otimes A^{\otimes(n-1)} \otimes A .
$$

The conclusion: we have

$$
H C .\left(I_{n !} E\right)=H_{\bullet}\left(\Lambda_{[n]}, E_{\#}^{[n]}\right)=H_{\bullet}\left(\Delta^{o p p}, \gamma_{n !} \gamma_{n}^{*} E_{n}^{\Delta}\right)=H_{\bullet}\left(\Delta^{o p p}, E_{n}^{\Delta} \otimes \gamma_{n !} k\right) \text {, }
$$

where we have used the projection formula (1.3) in the right-hand side. The homology of the category $\Delta^{o p p}$ can be computed by the standard complex; then by the Künneth formula, the right-hand side is isomorphic to

$$
H_{\bullet}\left(\Delta^{o p p}, E_{n}^{\Delta}\right) \otimes H_{\bullet}\left(\Delta^{o p p}, \gamma_{n !} k\right) \cong H_{\bullet}\left(\Delta^{o p p}, E_{n}^{\Delta}\right) \otimes H_{\bullet}\left(\Lambda_{[n]}, k\right) .
$$

By Lemma 2.1,

$$
H_{.}\left(\Delta^{o p p}, E_{n}^{\Delta}\right) \cong H H_{.}\left(A, E_{n}\right) \cong A^{\otimes n} .
$$

Since the category $\Lambda_{[n]}$ has an initial object $[n] \in \Lambda_{[n]}$, we have $k=i_{n ! k}$, so that the second multiple $H_{\text {. }}\left(\Lambda_{[n]}, k\right)$ is just $k$ in degree 0 .

The essential point of Proposition 4.9 is the following: the cyclic object $A_{\#}$ associated to an algebra $A$ inconveniently contains two things at the same time - the cyclic structure, which seems to be essential to the problem, and the bar resolution, which is needed only to compute the Hochschild homology $H H_{\text {. }}(A)$. Replacing $A_{\#}$ with the cyclic complex $L^{\bullet} \operatorname{tr}_{\#} A_{\#} \in$ $\mathcal{D}(\Lambda, k)$ disentagles these two. 
We note that while one still has to prove that this does not change the final answer, the construction itself looks pretty straightforward - if one wants to remove the non-essential bar resolution from the definition of the cyclic homology, Definition 4.8 seems to be the obvious thing to try. However, it was actually arrived at by a sort of a reverse engeneering process. To finish the section, perhaps it would be useful to show the reader the first stage of this process.

Assume given an associative algebra $A$, and fix a projective resolution $P$. of the diagonal $A$-module $A$. Then $H H_{\bullet}(A, M)$ can be computed by the complex

$$
\operatorname{tr}\left(P_{.}\right)=P_{\bullet} \otimes_{A^{o p p} \otimes A} A .
$$

How can one see the cyclic homology in terms of this complex? Or even simpler - what is the first differential in the spectral sequence (2.7), the Connes' differential $B: H H_{\bullet}(A) \rightarrow H_{\bullet+1}(A)$ ?

There is the following recepy which gives the answer. Let $\tau: P . \rightarrow A$ be the augmentation map. Consider the tensor product $P . \otimes_{A} P$. This is also a projective resotuion of $A$, and we actually have two natural quasiisomorphisms

$$
\tau_{1}, \tau_{2}: P_{\bullet} \otimes_{A} P_{\bullet} \rightarrow P_{.}
$$

given by $\tau_{1}=\tau \otimes \mathrm{id}, \tau_{2}=\mathrm{id} \otimes \tau$. These quasiisomorphisms are different. However, since both are maps between projective resolutions of the same object, there should be a chain homotopy between them. Fix such a homotopy $\iota: P_{.} \otimes_{A} P_{.} \rightarrow P_{\bullet+1}$.

Now we apply the trace functor $\operatorname{tr}$, and obtain two maps $\tau_{1}, \tau_{2}: \operatorname{tr}(P . \otimes$ $\left.P_{.}\right) \rightarrow \operatorname{tr}\left(P_{\bullet}\right)$, and a homotopy $\iota: \operatorname{tr}\left(P_{.} \otimes P_{\mathbf{.}}\right) \rightarrow \operatorname{tr}\left(P_{\bullet}+1\right)$ between them.

However, by the trace property of $\tau$, we also have an involution $\sigma$ : $\operatorname{tr}\left(P . \otimes_{A} P\right.$. $)$ which interchanges the two multiples. This involution obviously also interchages $\tau_{1}$ and $\tau_{2}$, but there is no reason why it should fix the homotopy $\iota$ - in fact, it sends $\iota$ to a second homotopy $\iota^{\prime}: \operatorname{tr}\left(P_{\bullet} \otimes_{A} P_{\bullet}\right) \rightarrow$ $\operatorname{tr}\left(P_{\bullet+1}\right)$ between $\tau_{1}$ and $\tau_{2}$.

The difference $\iota^{\prime}-\iota$ is then a well-defined map of complexes

$$
\iota^{\prime}-\iota: \operatorname{tr}\left(P_{\bullet} \otimes_{A} P_{\bullet}\right) \rightarrow \operatorname{tr}\left(P_{\bullet}+1\right) .
$$

On the level of homology, both sides are $H H_{.}(A)$; the map $\iota^{\prime}-\iota$ then induces exactly the Connes' differential $B: H H_{\bullet}(A) \rightarrow H H_{\bullet+1}(A)$.

To justify this recepy, we use Proposition 4.9 and identify $H C$. $(A)$ with $H C .\left(L^{\cdot} \operatorname{tr}_{\#}\left(A_{\#}\right)\right)$ rather than $H C .\left(A_{\#}\right)$. Then $L^{\bullet} \operatorname{tr}_{\#}\left(A_{\#}\right)$ is an object in 
$\mathcal{D}_{\text {const }}(\Lambda, k)$. Therefore, as noted in Remark 2.6, the Connes' differential $B$ only depends on the restriction of $L^{\bullet} \operatorname{tr}_{\#}\left(A_{\#}\right)$ to $\Lambda_{\leq 2} \subset \Lambda$. In other words, we do not need to compute the full $L^{\bullet} \operatorname{tr}_{\#}\left(A_{\#}\right)$ and to construct a full resolution $P^{\#}$ of the cyclic $A$-bimodule $A_{\#}$; it suffices to construct $P^{i}=P_{.}^{\#}([i])$ for $i=1,2$ (and then apply the functor $\operatorname{tr}$ ).

With the choices made above, we set $P^{1}=P_{\text {. }}$, and we let $P^{2}$ be the cone of the map

$$
P . \otimes P . \stackrel{(\tau \otimes \text { id }) \oplus(\text { id } \otimes \tau)}{\longrightarrow}(A \otimes P .) \oplus(P . \otimes A) .
$$

The involution $\sigma:[2] \rightarrow[2]$ acts on $P^{2}$ in the obvious way. We also need to define the transition maps $\iota_{f}$ for the two injections $d, d^{\prime}:[1] \rightarrow[2]$ and the two surjections $s, s^{\prime}:[2] \rightarrow[1]$. For $d_{1}$, the transition map $\iota_{d}: A \otimes P . \rightarrow P^{2}$ is the obvious embedding, and so is the transition map $\iota_{d^{\prime}}$. For the surjection $s$, we need a map $\iota_{s}$ from the cone of the map

$$
P . \otimes_{A} P . \stackrel{(\tau \otimes \mathrm{id}) \oplus(\mathrm{id} \otimes \tau)}{\longrightarrow} P . \oplus P .
$$

to $P_{.}$. On $P_{.} \oplus P_{\text {. }}$, the map $\iota_{s}$ is just the difference map $a \oplus b \mapsto a-b$; on $P . \otimes_{A} P_{\bullet}, \iota_{s}$ is our fixed homotopy $\iota: P_{\bullet} \otimes_{A} P_{\bullet} \rightarrow P_{\bullet+1}$. And similarly for the other surjection $s^{\prime}$.

We leave it to the reader to check that if one computes $\left.L^{\cdot} \operatorname{tr}_{\#}\left(A_{\#}\right)\right|_{\Lambda_{\leq 2}}$ using this resolution $P^{\#}$, then one obtains exactly (4.8) for the Connes' differential $B$.

\section{Discussion}

One of the most unpleasant features of the construction presented in Section 4 is the strong assumptions we need to impose on the tensor category $\mathcal{C}$. In fact, the category one would really like to apply the construction to is the category End $\mathcal{B}$ of endofunctors - whatever that means - of the category $\mathcal{B}$ of coherent sheaves on an algebraic variety $X$. But if $X$ is not affine, End $\mathcal{B}$ certainly does not have enough projectives, so that Example 4.5]does not apply, and it is unlikely that End $\mathcal{B}$ can be made homologically clean in the sense of Definition 4.4. We note that Definition 4.4 has been arranged so as not impose anything more than strictly necessary for the proofs; but in practice, we do not know any examples which are not covered by Example 4.5 .

As for the category End $\mathcal{B}$, there is an even bigger problem with it: while there are ways to define endofunctors so that $\operatorname{End} \mathcal{B}$ is an abelian category with a right-exact tensor product, it cannot be equipped with a right-exact 
trace functor tr. Indeed, it immediately follows from Definition 4.8 that the Hochschild homology groups $H H_{.}(\mathcal{C})$ of a tensor category $\mathcal{C}$ are trivial in negative homological degrees. If $\mathcal{C}=$ End $\mathcal{B}$, one of course expects $H H_{\text {. }}(\mathcal{C})=$ $H H$. $(X)$, the Hochschild homology $H H$. $(X)$ of the variety $X$, which by now is well-understood (see e.g. [W]). And if $X$ is not affine, $H H .(X)$ typically is non-trivial both in positive and in negative degrees. If $X$ is smooth and proper, $H H .(X)$ in fact carries a non-degenerate pairing, so that it is just as non-trivial in degrees $>0$ as in degrees $<0$. Thus the case of a non-affine algebraic variety is far beyond the methods developed in this paper.

The real reason for these difficulties is that we are dealing with abelian categories, while the theory emphatically wants to live in the triangulated world; as we explained in Example 2.5, even our main topic, cyclic bimodules, are best understood as objects of a triangulated category $\mathcal{D} \Lambda(\mathcal{C})$. Unfortunately, we cannot develop the theory from scratch in the triangulated context, since we do not have a strong and natural enough notion of an enhanced triangulated category (and working with the usual triangulated categories is out of the question because, for instance, the category of triangulated functors between triangulated categories is usually not a triangulated category itself). A well-developed theory would probably require a certain compromise between the abelian and the triangulated approach. We will return to it elsewhere.

Another thing which is very conspicously not done in the present paper is the combination of Section 4 and Section 3. Indeed, in Section 3, we are dealing with cyclic homology in the straightforward naive way of Section 2, and while we define the cyclic object $\widehat{A_{\#}}$ associated to a square-zero extension $\widetilde{A}$, we make no attempt to find an appropriate category $\operatorname{Sec}\left(\widehat{A-\operatorname{bim} o d_{\#}}\right)$ where it should live. This is essentially the reason why we cannot go further than square-zero extensions. At present, sadly, we do not really understand this hypothetical category $\operatorname{Sec}\left(\widehat{A-\text { bimod }_{\#}}\right)$.

One suspects that treating this properly would require studying deformations in a much more general context - instead of considering square-zero extensions of an algebra, we should look at the deformations of the abelian category of its modules, or at the deformations of the tensor category of its bimodules. This brings us to another topic completely untouched in the paper: the Hochschild cohomology $H H^{\bullet}(A)$.

Merely defining Hochschild cohomology for an arbitrary tensor category $\mathcal{C}$ is in fact much simpler than the definition of $H H_{.}(\mathcal{C})$, and one does not need a trace functor for this - we just set $H H^{\bullet}(\mathcal{C})=\operatorname{Ext}^{\bullet}(\mathrm{I}, \mathrm{I})$, where $\mathrm{I} \in \mathcal{C}$ is the unit object. However, it is well understood by now that just 
as Hochschild homology always comes equipped with the Connes' differential, the spectral sequence (2.7), and the whole cyclic homology package, Hochschild cohomology should be considered not as an algebra but as the socalled Gerstenhaber algebra; in fact, the pair $H H_{.}(-), H H^{\bullet}(-)$ should form a version of "non-commutative calculus", as proposed for instance in [TT]. Deformations of the tensor category $\mathcal{C}$ should be controlled by $H^{\bullet}(\mathcal{C})$, and the behaviour of $H H_{.}(\mathcal{C})$ and $H C .(\mathcal{C})$ under these deformations reflects various natural actions of $H^{\bullet}(-)$ on $H H_{\text {. }}(-)$.

We believe that a convenient development of the "non-commutative calculus" for a tensor category $\mathcal{C}$ might be possible along the same lines as our Section 4. Just as our category $\mathcal{D} \Lambda(\mathcal{C})$ is defined as the category of sections of the cofibration $\mathcal{C}_{\#} / \Lambda$, whose definition imitates the usual cyclic object $A_{\#}$, one can construct a cofibration $\mathcal{C}^{\#} / \Delta$ which imitates the standard cosimplicial object computing $H H^{\bullet}(A)$ - for any $[n] \in \Delta, \mathcal{C}^{\#}([n])$ is the category of

polylinear right-exact functors from $\mathcal{C}^{n-1}$ to $\mathcal{C}$, and the transition functors between various $\mathcal{C}^{\#}([n])$ are induced by the tensor product on $\mathcal{C}$. Then one can define a triangulated category $\mathcal{D} \Delta(\mathcal{C})$, the subcategory in $\mathcal{D}\left(\operatorname{Sec}\left(\mathcal{C}^{\#}\right)\right)$ of complexes with cocartesian homology; the higher structures on $\mathrm{HH}^{\bullet}(\mathcal{C})$ should be encoded in the structure of the category $\mathcal{D} \Delta(\mathcal{C})$, and relations between $H H_{.}(\mathcal{C})$ and $H H^{*}(\mathcal{C})$ should be reflected in a relation between $\mathcal{D} \Lambda(\mathcal{C})$ and $\mathcal{D} \Delta(\mathcal{C})$. We will proceed in this direction elsewhere. At present, the best we can do is to make the following hopeful observation:

- the category $\operatorname{Sec}_{\text {cart }}\left(\mathcal{C}^{\#}\right)$ is naturally a braided tensor category over $k$.

The reason for this is very simple: if one writes out explicitly the definition of $\operatorname{Sec}_{\text {cart }}\left(\mathcal{C}^{\#}\right)$ along the lines of Lemma 2.2, one finds out that it coincides on the nose with the Drinfeld double of the tensor category $\mathcal{C}$.

\section{References}

[BD] I. Bucur and A. Deleanu, Introduction to the theory of categories and functors, Interscience Publication John Wiley \& Sons, Ltd., LondonNew York-Sydney 1968.

[C1] A. Connes, Non-commutative differential geometry, I, II, preprint IHES oct. 1982, may 1983.

[C2] A. Connes, Cohomologie cyclique et foncteur Ext ${ }^{n}$, Comptes Rendues Ac. Sci. Paris Sér. A-B, 296 (1983), 953-958. 
[FT] B. Feigin and B. Tsygan, Additive K-Theory, in Lecture Notes in Math. 1289 (1987), 97-209.

[GM1] S. Gelfand and Yu. Manin, Methods of homological algebra, Nauka Publishers, Moscow, 1988 (in Russian).

[GM2] S. Gelfand and Yu. Manin, Homological algebra, Itogi vol. 38 (Algebra V), VINITI, Moscow, 1989 (in Russian).

[Ge] E. Getzler, Cartan homotopy formulas and the Gauss-Manin connection in cyclic homology, in Quantum deformations of algebras and their representations (Ramat-Gan, 1991/1992; Rehovot, 1991/1992), Israel Math. Conf. Proc. 7, Bar-Ilan Univ., Ramat Gan, 1993, 65-78.

[Go] Th. Goodwillie, Cyclic homology, derivations, and the free loopspace, Topology 24 (1985), 187-215.

[Gr] A. Grothendieck, Expose VI: Catégories fibré et descente, in SGAI: Revétements étales et groupe fondamental, Lecture Notes in Math., 224, Springer, Berlin; 145-194.

[Ka] D. Kaledin, Non-commutative Hodge-to-de Rham degeneration via the method of Deligne-Illusie, math.KT/0611623.

[L] J.-L. Loday, Cyclic Homology, second ed., Springer, 1998.

[TT] D. Tamarkin and B. Tsygan, The ring of differential operators on forms in noncommutative calculus, in Graphs and patterns in mathematics and theoretical physics. Proc. Sympos. Pure Math. 73, AMS, Providence, RI, 2005; 105-131.

[Ts] B. Tsygan, Homology of Lie algebras over rings and Hochshild homology, Uspekhi Mat. Nauk, 38 (1983), 217-218.

[W] C. Weibel, Cyclic homology for schemes, Proc. AMS 124 (1996), 1655-1662.

Steklov Math Institute

Moscow, USSR

E-mail address: kaledin@mccme.ru 